\title{
Puerta de Entrada al Consumo de Sustancias Ilegales en Colombia: Infracciones a la Norma de Inicio
}

\section{The gateway to illegal substance use in Colombia: violations of the gateway rule}

Jose A. Posada-Villa, Edwin Herazo y Adalberto Campo-Arias

Instituto de Investigación del Comportamiento Humano, Bogotá, Colombia. campoarias@comportamientohumano.org

Recibido 8 Agosto 2008/Enviado para Modificación 19 Marzo 2009/Aceptado 20 Abril 2009

\section{RESUMEN}

Objetivo Establecer la frecuencia de la infracción a la norma del inicio de sustancias ilegales con el uso de alcohol o nicotina en adultos colombianos de la población general.

Métodos Se realizó un estudio descriptivo con adultos de la población general de Colombia. Se determinaron las frecuencias de inicio de consumo de sustancias ilegales con sustancias distintas a alcohol o nicotina.

Resultados En un total de 4426 adultos participaron en la investigación y se observó que 127 personas $(3,3 \%)$ iniciaron el consumo de sustancias ilegales con el uso de sustancias diferentes a alcohol o nicotina: $2,3 \%$ otra sustancia ilegal antes que marihuana; 0,6 \% marihuana antes que alcohol o nicotina; y 0,4\% otra sustancia ilegal antes que alcohol o nicotina.

Conclusiones Un número reducido de consumidores de sustancias ilegales en Colombia inicia con sustancias distintas a alcohol o nicotina. Es necesario investigar los factores asociados con este inicio.

Palabras Clave: Trastornos relacionados con sustancias, epidemiología, prevalencia, población adulta, salud pública (fuente: DeCS, BIREME).

\section{ABSTRACT}

Objective Establishing the frequency of gateway illegal substance use with alcohol or nicotine being violated amongst Colombian adults in the general population.

Methods A descriptive study was carried out on adults from the general Colombian population. Gateway frequencies were determined for illegal substance use regarding substances other than alcohol or nicotine.

Results A total of 4,426 adults participated in this research. A group of 127 people (3.3\%) reported that they had started illegal substance use before using alcohol or nicotine; $2.3 \%$ of them had used other illegal substance before using cannabis, 0,6\% had smoked cannabis before using alcohol or nicotine and $0,4 \%$ had used other illegal substances before using alcohol or nicotine. 
Conclusions A small group of people who use illegal substances in Colombia start with substances different to alcohol or nicotine. The factors associated with gateway use of illegal substances (regarding substance other than alcohol or nicotine) should be investigated.

Key Words: Substance-related disorders, epidemiology, prevalence, adult population, public health (source: MeSH, NLM).

$\mathrm{E}$ 1 consumo de sustancias que pueden inducir dependencia representa un problema de salud pública (1). En Colombia, alrededor del $20 \%$ de los adultos consume nicotina (fumadores regulares), el $17 \%$ está en alto riesgo de depencencia a alcohol, el $4 \%$ ha consumido marihuana alguna vez en la vida y un $2 \%$ ha usado alguna otra sustancia ilegal durante la vida (2).

Generalmente, el consumo de sustancias ilegales se inicia durante la adolescencia (3). En el mayor número de casos, los adolescentes experimentan con sustancias de fácil acceso y más socialmente aceptadas como alcohol y nicotina (4). En consecuencia, alcohol o nicotina son las sustancias que sirven de puerta de entrada al consumo de sustancias ilegales (5). Sin embargo, en algunos casos el consumo de sustancias ilegales se hace con el uso de sustancias distintas a alcohol o nicotina, infracción a la norma de inicio (6-9). Los estudios disponibles más recientes muestran que aproximadamente el $5 \%$ de los consumidores de sustancias ilegales infringen la norma de inicio con alcohol o nicotina $(6,7)$.

La sustancia que sirve de puerta de entrada al consumo de sustancias ilegales tiene connotaciones especiales. Las personas que inician ese consumo sin consumo previo de alcohol o nicotina presentan posteriormente un riesgo mayor de reunir criterios para dependencia (10) y dificultad para el abandono definitivo de la misma (11). Esto se asocia a más deterioro en el funcionamiento global, en la calidad de vida y riesgo de muerte por diferentes causas (12). Los datos disponibles sugieren que el inicio con otra sustancia diferente a alcohol o nicotina representa un indicador de una condición clínica más grave con peor respuesta a las intervenciones terapéuticas (13).

Hasta la fecha, no se ha investigado la puerta de entrada al consumo de sustancias ilegales y la infracción a la norma de inicio por alcohol o nicotina en población adulta de Colombia. El presente informe es un análisis secundario del estudio nacional de salud mental de Colombia de 2003 y el objetivo del mismo es presentar las infracciones a la norma general de inicio de consumo de sustancias ilegales mediante el uso de sustancias diferentes a alcohol o nicotina. 


\section{MÉTODOS}

El presente es un estudio descriptivo que hace parte del estudio nacional de salud mental y la Encuesta Mundial de Salud Mental. El Comité de Ética de la Fundación FES Social revisó y aprobó el proyecto de investigación. Todos los participantes firmaron consentimiento informado. Para la investigación se siguieron los principios éticos para la investigación con seres humanos delineados en la Declaración de Helsinki (14) y las normas colombianas para la investigación en salud (15).

\section{Muestra}

Se tomó una muestra probabilística multietápica y estratificada de la población de hogares colombianos de la Muestra Maestra del Ministerio de la Protección Social (16). Esta muestra tiene un diseño para el uso en estudios epidemiológicos nacionales. Se incluyeron adultos entre 18 y 65 años de edad residentes en el área urbana de 60 municipios del país. Asimismo, se estimó la distribución de los estratos en los municipios de la muestra maestra en función del tamaño de los estratos correspondientes. Para este proceso se utilizaron las proyecciones de población municipal urbana a partir del censo del Departamento Administrativo Nacional de Estadísticas (DANE) para el año 2003 (17).

Se necesitaba la participación efectiva de 4596 adultos para cumplir el requisito mínimo exigido para la Encuesta Mundial de Salud Mental para Colombia. Las entrevistas efectivas eran las que cumplían los requisitos de calidad pedidos por la Universidad de Harvard y la Organización Mundial de la Salud: se vigiló continuamente la proporción de respondientes que contestaron afirmativamente la sección de tamizaje para los diferentes diagnósticos incluidos por cada entrevistador, la tasa de respuesta por sexo, para detectar oportunamente probables problemas en el patrón de respuesta de los respondientes. Finalmente, estadísticamente se verificó el control de calidad mediante la identificación de posibles errores e inconsistencias en relación con los tiempos de los eventos (inicio, períodos en los que estaban presentes, consistencia de las edades, primera y última fecha de utilización de los servicios), respuestas faltantes e introducir los valores correctos cuando ello era posible. Se estimó una participación de 5750 hogares con el supuesto de una participación máxima efectiva del $70 \%$, sin substitución de los residentes originalmente seleccionados si no podía llevar a cabo la entrevista.

La entrevista realizada se dividía en dos partes para evitar la sobrecarga de los participantes. La primera incluía el diagnóstico y la segunda, información sobre detalles de los trastornos identificados en la primera parte. Todas las personas que 
reunieron criterios para un trastorno completaron la segunda parte. Además, se tomó al azar un $25 \%$ adicional de las personas que no reunieron criterios para trastorno mental para completar la segunda parte de la entrevista.

\section{Instrumento}

Se usó la versión 15 para adultos de la Entrevista Diagnóstica Internacional Compuesta (Composite Internacional Diagnostic Interview, CIDI, en inglés). La Organización Mundial de la Salud diseñó esta entrevista en cooperación con la Administración de Alcohol, Abuso de Drogas y Salud Mental de los Estados Unidos (Alcohol Drug Abuse and Mental Health Administration, en inglés). La entrevista permite identificar 17 trastornos mentales principales y seis secundarios, con la inclusión de consumo de sustancias, durante la vida, en los últimos 12 meses y en los últimos 30 días según criterios diagnósticos del DSM-IV y el CIE-10. De la misma forma, para el diagnóstico en los últimos 12 meses permite establecer la severidad según sea grave, moderado o leve y el deterioro en el funcionamiento global (18).

\section{Procedimiento}

Para la aplicación de la entrevista se entrenaron 11 supervisores locales y 39 encuestadores que hacían entrevistas cara a cara en los hogares con el uso de la CAPI. Se siguieron los lineamientos de la Organización Mundial de la Salud para la adaptación cultural de las preguntas, es decir, que conservaran el mismo sentido lingüístico(18).

Análisis estadístico

La Universidad de Harvard y la Organización Mundial de la Salud aprobaron la base de datos para este análisis. Se estableció el consumo de sustancia a lo largo de la vida y se estableció la prevalencia de cada una de las infracciones a la norma de inicio de sustancias ilegales mediante uso de sustancias distintas a alcohol o nicotina en toda la muestra participante y en cada uno de los grupos de consumidores (19).

\section{RESULTADOS}

Un total de 4426 adultos respondieron la primera parte de la entrevista, de las cuales 2422 personas $(54,7 \%)$, igualmente, completaron la segunda parte de la misma. La población por edad se distribuyó en los siguientes rangos: entre 18 y 34 años $47,7 \%$; entre 35 y 49 años, 32,9 \%; y entre 50 y 65 años, 19,4 \%. El 56,2 \% de los participantes eran mujeres, el 55,6\% se encontraban casados y el 53,2 \% 
eran empleados y 25,1\% amas de casa. La distribución por nivel de educación fue: primaria completa o menos $30,3 \%$; secundaria completa o incompleta, $44,5 \%$; y 12 años o más de escolaridad formal, $25,2 \%$.

Un 93,6\% de los entrevistados informó consumo de alcohol alguna vez en la vida; $47,6 \%$, consumo de nicotina; $9,3 \%$, consumo de marihuana; $3,5 \%$, consumo de cocaína; y 5,3\%, consumo de otras sustancias. En las Tablas 1 y 2 se presentan las infracciones a la norma de inicio por alcohol o nicotina.

Tabla 1. Prevalencia de cada violación en una muestra de 4426 personas de la población general de Colombia

\begin{tabular}{lcc}
\hline Violación & Frecuencia & $\%$ \\
\hline Marihuana antes de alcohol y tabaco & 25 & 0,6 \\
Otra sustancia ilícita antes de alcohol y tabaco & 19 & 0,4 \\
Otra sustancia ilícita antes de marihuana & 102 & 2,3 \\
Cualquiera de las anteriores & 127 & 3,3 \\
\hline
\end{tabular}

Tabla 2. Prevalencia de cada violación en cada consumidor de la población

\begin{tabular}{|c|c|c|}
\hline Consumidor de alcohol ( $n=4$ 143) & Frecuencia & $\%$ \\
\hline Marihuana antes de alcohol y tabaco & 24 & 0,6 \\
\hline Otra sustancia ilicita antes de alcohol y tabaco & 18 & 0,4 \\
\hline Otra sustancia ilicita antes de marihuana & 101 & 2,4 \\
\hline Cualquiera de las anteriores & 125 & 3,4 \\
\hline \multicolumn{3}{|l|}{ Consumidor de tabaco $(n=2$ 106) } \\
\hline Marihuana antes de alcohol y tabaco & 17 & 0,8 \\
\hline Otra sustancia ilicita antes de alcohol y tabaco & 9 & 0,4 \\
\hline Otra sustancia ilicita antes de marihuana & 65 & 3,1 \\
\hline Cualquiera de las anteriores & 82 & 4,3 \\
\hline \multicolumn{3}{|l|}{ Consumidor de marihuana $(n=413)$} \\
\hline Marihuana antes de alcohol y tabaco & 25 & 6,0 \\
\hline Otra sustancia ilicita antes de alcohol y tabaco & 17 & 4,1 \\
\hline Otra sustancia ilícita antes de marihuana & 19 & 4,6 \\
\hline Cualquiera de las anteriores & 44 & 14,8 \\
\hline \multicolumn{3}{|l|}{ Consumidor de cocaína ( $n=157)$} \\
\hline Marihuana antes de alcohol y tabaco & 6 & 3,8 \\
\hline Otra sustancia ilicita antes de alcohol y tabaco & 8 & 5,1 \\
\hline Otra sustancia ilícita antes de marihuana & 36 & 22,9 \\
\hline Cualquiera de las anteriores & 42 & 31,8 \\
\hline \multicolumn{3}{|l|}{ Consumidor de otras $(n=236)$} \\
\hline Marihuana antes de alcohol y tabaco & 6 & 2,5 \\
\hline Otra sustancia ilicita antes de alcohol y tabaco & 19 & 8,0 \\
\hline Otra sustancia ilicita antes de marihuana & 102 & 43,2 \\
\hline Cualquiera de las anteriores & 108 & 53,8 \\
\hline
\end{tabular}

\section{DISCUSIÓN}

En el presente estudio se observa que un número reducido de adultos colombianos de la población general iniciaron el consumo de sustancias ilegales mediante el uso de sustancias diferentes a alcohol o nicotina. 
Los resultados de esta investigación son concordantes con la mayoría de los estudios disponibles que informan que el mayor número de consumidores de sustancias ilegales usan habitualmente como puerta de entrada alcohol o nicotina. Golub et al. observaron que el $94 \%$ de las personas iniciaron con alcohol o tabaco, que el $26 \%$ de los consumidores de alcohol o tabaco experimentaron posteriormente con marihuana y que el $29 \%$ de los consumidores de marihuana usaron otra sustancia ilegal posteriormente (6). Por su parte, Ried et al. encontraron que en el más alto porcentaje el consumo de alcohol o marihuana precede el uso de sustancias ilegales como cocaína y metanfetamina y que la marihuana sólo influenciaba el uso posterior de heroína (7).

La puerta de entrada al consumo de sustancias ilegales se relaciona con un número importante de variables individuales y contextuales. Por ejemplo, en un estudio se observó que en adolescentes residentes en la calle es más frecuente el inicio de consumo de sustancias con el uso de una sustancia distinta a alcohol o nicotina (20). En otra investigación se encontró que la progresión en el consumo de sustancia guarda relación con factores ambientales. Se observó que en las generaciones más jóvenes era más probable que se iniciara el consumo de sustancias ilegales con marihuana y pasara de este consumo al uso de una sustancia pesada (8).

Las personas que inician el consumo de sustancias ilegales con la infracción de la norma de inicio por alcohol o nicotina muestran una prevalencia incrementada para otros trastornos mentales que aquellas que inician con las sustancias habituales (alcohol, nicotina o marihuana) $(21,22)$. Igualmente, este grupo de personas muestran mayor riesgo de reunir criterios para dependencia a cualquier sustancia, tanto legal como ilegal (23). Esto puede sugerir que la sustancia que sirve de puerta de entrada al consumo puede ser un indicador temprano de psicopatología (24).

El inicio de consumo de sustancia en población adulta colombiana con el uso de las sustancias que habitualmente sirve de puerta de entrada, alcohol, nicotina o marihuana, sugieren la necesidad de centrar esfuerzos en la prevención del consumo de sustancias legales como alcohol y nicotina. La reducción en el inicio del consumo de estas sustancias puede reflejarse en el menor consumo de sustancias ilegales que producen a corto plazo un mayor impacto negativo en la vida de los consumidores $(7,25)$.

La principal fortaleza de este estudio fue contar con una muestra representativa de la población adulta colombiana y valorar el consumo de sustancias mediante una entrevista estructurada. Sin embargo, posee las limitaciones propias de un 
trabajo descriptivo e indagar comportamientos sensibles, como el consumo de sustancias ilegales.

Se concluye que la mayoría de los consumidores adultos de sustancias ilegales en Colombia usan inicialmente sustancias tradicionales (alcohol o nicotina). Es necesario explorar factores asociados al no cumplimiento de la tendencia de iniciarse en el consumo de sustancias ilegales mediante la experimentación con alcohol o nicotina

Conflicto de intereses: ninguno

\section{REFERENCIAS}

1. Brugal MT, Rodríguez-Martos A, Villalbí JR. Nuevas y viejas adicciones: implicaciones para la salud pública. Gac Sanit 2006; 20 (supl. 1): 55-62.

2. Posada JA, Torres Y. Estudio nacional de salud mental y consumo de sustancias psicoactivas, Colombia, 1993. Santafé de Bogotá: Ministerio de Salud; 1995.

3. Roldan C. Abuso de sustancias. Propuestas preventivas, detección precoz y abordaje terapéutico. Arch Argent Pediatr 2003; 101: 1-11.

4. Medina-Mora ME, Natera G, Borges G, Craviato P, Fleiz C, Tapia-Conyer R. Del siglo XX al tercer milenio. Las adicciones y la salud pública: drogas, alcohol y sociedad. Salud Mental 2001; 24: 3-19.

5. Kandel D, Yamaguchi K. Testing the gateway hypothesis. Addiction 2006; 101: 470-2.

6. Hall W, Lynskey M. Is cannabis a gateway drug? Testing hypotheses about the relationship between cannabis use and the use of other illicit drug. Drug Alcohol Rev 2005; 24: 39-48.

7. Golub A, Johnson BD. The misuse of the 'gateway theory' in us policy on drug abuse control: a secondary analysis of the muddled deduction. Int J Drug Policy 2002; 13: 5-19.

8. Rieid L, Elifson K, Sterk C. Ecstasy and gateway drugs: Initiating the use of ecstasy and other drugs. Ann Epidemiol 2007; 17: 74-80.

9. Golub A, Johnson BD. Variation in youthful risks of progression from alcohol and tobacco to marijuana and to hard drugs across generations. Am J Public Health 2001; 91: 225-32.

10. Morral A, McCaffrey D, Paddock S. Reassessing the marijuana gateway effect. Addiction 2002; 97: 1493-504.

11. Lynskey MT, Heath AC, Bucholz KK, Slutske WS, Madden PAF, Nelson EC, et al. Escalation of drug use in early-onset cannabis users vs co-twin control. JAMA 2003; 289: 427-33.

12. Sussman S, Dent CW. Five-year prospective prediction of self-initiated quitting of cigarette smoking of high-risk youth. Addict Behav 2007; 32: 1094-8.

13. Kertesz S, Pletcher M, Safford M, Halanych J, Kirk K, Schumacher J, et al. Illicit drug use in young adults and subsequent decline in general health: The Coronary Artery Risk Development in Young Adults (CARDIA) Study. Drug Alcohol Depend 2003; 88: 22433.

14. World Medical Association [Internet]. Declaración de Helsinki. Disponible en: http:// www.wma.net/e/policy/b3.htm Consultado:29-03-2008.

15. Ministerio de Salud de Colombia. Resolución 008430 por la cual se establecen las normas científicas, técnicas y administrativas para la investigación en salud. Bogotá; 1993. 
16. Muestra Maestra para Estudios en Salud. Bogotá: Ministerio de la Protección Social. Dirección de Salud Pública; 2003.

17. Encuesta Nacional de Hogares. Bogotá: Departamento Administrativo Nacional de Estadística (DANE); 2003.

18. Kessler RC, Ustun TB. The World Mental Health (WMH) Survey Initiative Version of the World Health Organization (WHO) Composite International Diagnostic Interview (CIDI). Int J Methods Psychiatr Res 2004; 13: 93-121.

19. Moreno-Altamirano A, López-Moreno S, Corcho-Berdugo A. Principales medidas en epidemiologia. Rev Salud Publica Mex 2000; 42: 337-48.

20. Ginzler J, Cochran B, Domenech-Rodriguez M, Cauce A, Whitbeck L. Sequential progression of substance use among homeless youth: an empirical investigation of the gateway theory. Substance Use Misuse 2003; 38: 725-58.

21. Farrell M, Howes S, Bebbington P, Brugha T, Jenkins R, Lewis G, et al. Nicotine, alcohol and drug dependence and psychiatric comorbidity. Results of national household survey. Br J Psychiatry 2001; 179: 432-7.

22. Martínez-Ortega JA, Jurado D, Martínez-González MA, Gurpegui M. Nicotine dependence, use of illegal drugs and psychiatric morbidity. Addict Behav 2006; 31: 1722-9.

23. Breslau N, Petersen EL. Smoking cessation in young adults: age at initiation of cigarette smoking and other suspected influences. Am J Public Health 1996; 86: 214-20.

24. Degenhardt L, Coffey C, Moran P, Carlin JB, Patton GC. The predictors and consequences of adolescent amphetamine use: findings from the victorian adolescent health cohort study. Addiction 2007; 102: 1076-84.

25. Sun W, Skara S, Sun P, Dent CW, Sussman S. Project towards no drug abuse: long-term substance use outcome evaluation. Prev Med 2006; 42: 188-92. 